\title{
Stability of Palm Oil-based Emulsion Liquid Membrane for Succinic Acid Extraction from Aqueous Solution
}

\author{
N. Jusoh ${ }^{1}$, N. Othman ${ }^{2 *}$ \\ ${ }^{1}$ Department of Chemical Engineering, Faculty of Chemical and Energy Engineering, \\ Universiti Teknologi Malaysia, 81310 UTM Johor Bahru, Johor, Malaysia \\ ${ }^{2}$ Centre of Lipids Engineering and Applied Research (CLEAR), Ibnu Sina Institute of \\ Scientific and Industrial Research, Universiti Teknologi Malaysia, 81310 UTM Johor \\ Bahru, Johor, Malaysia
}

\begin{abstract}
Emulsion liquid membrane (ELM) process has high potential in the separation of succinic from the fermentation broth. However, the major drawback of this technology is the stability of emulsion globules during the extraction process and the chemical involved in the liquid membrane formulation. This study investigate the stability of ELM using a greener formulation containing Amberlite LA-2 as a carrier, Span 80 and Tween 80 as a surfactant, palm oil as a diluent and sodium carbonate $\left(\mathrm{Na}_{2} \mathrm{CO}_{3}\right)$ as an aqueous stripping agent. The emulsion stability was evaluated by observing the water-oil separation of the emulsion and microscopic image of emulsion droplets count and size. Several operating parameters including the organic to internal ratio, homogenizer speed, homogenizing time, and surfactant concentration, and surfactant blend were investigated. The results show the most stable waterin-oil emulsion was observed at 3:1 organic to internal ratio; 7000rpm homogenizer speed; 5 minute emulsification time; $3 \%(\mathrm{w} / \mathrm{v})$ surfactant at HLB 8 . Besides, the extraction study shows $70 \%$ of the succinic acid was extracted at $0.01 \mathrm{M} \mathrm{Na}_{2} \mathrm{CO}_{3}, 1: 3$ treat ratio, and $0.7 \mathrm{M}$ Amberlite in palm oil at optimum primary emulsion stability conditions. This indicates the potential of using palm oil based ELM for the extraction of succinic acid.
\end{abstract}

Keywords: Succinic acid, emulsion liquid membrane, palm oil, primary emulsion, stability

\subsection{INTRODUCTION}

Succinic acid (IUPAC name: butanedioic acid) can be applied in many industrial applications as commodity and specialty chemical. For example, succinic acid can derive many important intermediate chemicals such as N-methyl pyrrolidinone, 1,4-butanediol, $\gamma$ butyrolactone, and tetrahydrofuran [14]. On the other hand, uses of succinic acid in specialty chemical are rapidly growing such as for food ingredients, feed additives, plant growth stimulants, and health agents [1, 5-8].

Recently, biological production of succinic acid from abundant and available biomass has become a topic of worldwide interest [9-13]. However, the biological production of succinic acid is still not economically competitive compared to petrochemical production route. This is because of low product concentration in the fermentation broth and difficulty in purification process [14]. In addition, existence of by-product complicates the product purification.

Many methods have been proposed to recover succinic acid from fermentation broth such as precipitation [15], direct crystallization [16], membrane filtration [17], and solvent extraction $[18,19]$. 
The disadvantage of precipitation is large dosage of $\mathrm{Ca}(\mathrm{OH})_{2}$ required to precipitate succinic acid in fermentation broth, which lead to high operation cost. Besides that, further treatments are needed to purify calcium succinate formed. On the other hand, direct crystallization might provide the desired product (in solid or crystal form), without the need for many unit operations. However, the product yield is low because much succinate is still residual in broth. Opposite from that, membrane filtration provides high purity of product, but constraint by high cost of device and also membrane pollution which leads to high operation cost. Solvent extraction offer some advantages such as high output and low energy consumption [20]. Unfortunately, the extraction process requires large quantities of extraction agent.

One of promising methods for succinic acid recovery is emulsion liquid membrane (ELM). ELM is a system where a thin liquid film of organic reagent divides the aqueous external feed and internal product phases. The solute of interest in the feed phase reacts with organic carrier at the external interface and migrates across the organic membrane to the internal interface. At the internal interface, stripping solution strip the solute and the carrier migrates back to the external interface, and the extraction continues.

ELM process has received considerable attention because of their potential advantages over other separation process, particularly over distillation, solvent extraction, and separation by solid membrane [21-26]. ELM fulfills the promise of providing high transport efficiencies due to high interfacial area for mass transfer, high transfer flux with incorporation of carrier, economical, low energy consumption, extraction and stripping in a single stage, efficient for low solute concentration, and low solvent requirement.

However, the main drawback related to ELM is the emulsion instability. This can be attributed to the emulsion formulation in terms of the choice of carrier, diluent, surfactant, stripping agent, and emulsification procedure. The carrier should be selective to the target succinic acid solute while the stripping agent and the type of surfactant must be properly chosen to minimize the water transport during extraction process.

The diluent in ELM process is very important, since it is the major component of the membrane phase and is crucial for emulsion stability. Diluent should have a low solubility in water in order to create the membrane phase, it should also provide high carrier solubility, have a high-boiling point, be non-toxic and relatively cheap [27, 28]. Most studies have commonly used kerosene as organic diluent due to its viscosity, readily availability and non-polar character. However, kerosene is not considered environmentally friendly and harmful to human. Alternatively, palm oil can be chosen as renewable organic diluent, as it is readily available and may contain natural surface-active agents, which improve the stability of an emulsion [29]. In addition, palm oil was proven to work well in extraction of heavy metal and phenol [30, 31].

In this study, the main focus was to investigate primary water-in-oil emulsion stability using Amberlite LA2 as carrier, palm oil as diluent, sodium carbonate as aqueous stripping agent, Span 80 and Tween 80 as surfactant. Several operating parameters were investigated including organic to internal ratio, homogenizer speed, homogenizing time, surfactant concentration, and surfactant blend. 
Besides, the most stable emulsion was then being used to extract succinic acid. The performance was evaluated by a few parameters which are stripping agent concentration, carrier concentration and volume ratio of emulsion to external phase (treat ratio).

\subsection{METHODS}

\subsection{Materials}

Palm oil (cooking oil) as diluent produced by Lam Soon Edible Oils. Amberlite LA-2 as carrier was obtained from Merck. Amberlite LA-2 used was a mixture of straight chain secondary amine $(\mathrm{M}=374 \mathrm{~g} / \mathrm{mol})$. Solid sodium carbonate $\left(\mathrm{Na}_{2} \mathrm{CO}_{3}\right)$ (99.9\% assay) for internal stripping reagent was also obtained from Merck. Meanwhile, sorbitan monooleate (Span 80) (with more than $60 \%$ oleic acid composition), and polyoxyethylene sorbitan monooleate (Tween 80) (contain more than $58 \%$ oleic acid) as surfactant was purchased from Sigma Aldrich. All these reagents and solutions were used directly as received without further purification.

\subsection{Stability of Primary Emulsion}

Organic liquid membrane solution containing Amberlite LA2, Span 80 and Tween 80 in palm oil and aqueous $\mathrm{Na}_{2} \mathrm{CO}_{3}$ solution (1 to 3 organic to internal ratio) was emulsified at different speeds $(5000 \mathrm{rpm}$ to 12000 $\mathrm{rpm}$ ) for different times (3 to 20 minutes) using motor driven homogenizer (Heidolph Silent Crusher M) to obtain water-in-oil primary emulsion. Immediately after emulsification, the emulsion was poured into a $10 \mathrm{~mL}$ measuring cylinder. The stability of the emulsion was determined by recording the volume of aqueous phase separated as a function of time. More aqueous phase separated indicate the emulsion is unable to remain dispersed and less stable. The emulsification conditions for each investigation are given in Table 1. Note that effect of surfactant blend was varied by varying the composition of Span 80 and Tween 80 according to the hydrophilic-lipophilic balance (HLB) using Equation 1.

$\%(A)=\frac{\left(H L B_{A B}-H L B_{B}\right)}{H L B_{A}-H L B_{B}} x 100 \%$

where $\%(A)$ is the composition of Tween 80 in surfactant mixture, $H L B_{A B}$ is the HLB of mixture of Span 80 and Tween 80, and $A$ and $B$ represents Tween 80 and Span 80 respectively.

The aggregation of the emulsion droplets was directly observed under a polarized microscope (Olympus CX31). All the images were captured approximately at 10 minutes after the homogenizing process. Meanwhile, number of droplets formed was counted within circle of $30 \mu \mathrm{m}$ radius and size of droplet was determined by taking average size of 30 droplets using VImage 2014 software. The size of the droplet was expressed as Sauter mean diameter $\left(\mathrm{d}_{32}\right)$, defined in Equation 2:

$d_{32}=\frac{\sum\left(n_{i} \cdot d_{i}^{3}\right)}{\sum\left(n_{i} \cdot d_{i}^{2}\right)}$

\subsection{Extraction Study}

The prepared primary emulsion was dispersed into the $50 \mathrm{~mL}$ beaker containing $30 \mathrm{~mL}$ of $40 \mathrm{~g} / \mathrm{L}$ succinic solution as a feed phase to form waterin-oil in water emulsion. The concentration was based on the concentration of practical fermentation broth [32]. The mixture of water-in-oil in water emulsion was agitated using a motor stirrer with an extraction speed 
Table 1 Emulsification condition for investigating primary emulsion stability

\begin{tabular}{ll}
\hline Emulsification Parameters & Range \\
\hline Effect of organic to internal ratio (O:I) & $1: 1,2: 1,3: 1$ \\
Effect of homogenizer speed (rpm) & $5000,7000,9000,12000$ \\
Effect of homogenizing time (min) & $3,5,10,15,20$ \\
Effect of surfactant concentration (\%w/v) & $1,3,5,7,10$ \\
Effect of HLB (value) of the surfactant mixture & $4.3,5,6,7,8$ \\
\hline
\end{tabular}

of $300 \mathrm{rpm}$ for 3 minutes. Then the mixtures were separated by pouring into the separation funnel and leave about half an hour for phase separation. The external aqueous phase was analyzed using high performance liquid chromatography (HPLC) with an ion exchange column (Aminex HPX-87H, $300 \mathrm{~mm} \times 7.8 \mathrm{~mm}$, Bio$\mathrm{Rad})$ and $0.005 \mathrm{M} \mathrm{H}_{2} \mathrm{SO}_{4}$ as mobile phase. The extraction performance of succinic acid was evaluated using Equation 3.

$$
\begin{aligned}
& \text { Extraction }(\%) \\
& =\frac{[S A]_{i(a q)}-[S A]_{f(a q)}}{[S A]_{f(a q)}} \times 100
\end{aligned}
$$

where $[S A]_{i(a q)}$ is the initial acid concentration in external aqueous phase $(\mathrm{g} / \mathrm{L}) ;[S A]_{f(a q)}$ is the acid concentration in external aqueous phase after extraction $(\mathrm{g} / \mathrm{L})$.

\subsection{RESULTS AND DISCUSSION}

\subsection{Stability Study}

\subsubsection{Effect of Organic Phase to Internal Phase Ratio}

The effect of organic to internal phase ratio is demonstrated in Figure 1. Phase ratio of the emulsion was varied at $1: 1,2: 1$, and $3: 1$ while the volume of the emulsion was kept constant at 10 ml. 1:1 ratio shows the most unstable emulsion, where $26 \%$ of aqueous phase was separated in 10 minutes, which indicates the internal aqueous phase did not remained dispersed in the emulsion. This is due to the inherent nature of palm oil with the accompanying stearin as natural surfactant in the oil which alters the composition of Span 80 at the oilwater interface and reduces dispersion of internal phase in the organic phase, hence affects its stability. The result is in line with other studies conducted by McClements et al. [33] and Chow and Ho [29] which stated that the adsorption of stearin at the water-oil interface would certainly affect the stability. In addition, microscopic image of the emulsion at different organic to internal ratio is shown in Figure 2. Ratio 1:1 formed less droplets compared to $2: 1$ and $3: 1$, showing that low dispersion of internal phase will reduce the emulsion stability. Meanwhile, increasing the ratio to $2: 1$ and $3: 1$ will increase the emulsion stability due to the increasing of Span 80 composition at the interface, which reduces more interfacial tension, thus resulting in more droplets formed. Besides, increasing organic fraction also increase membrane phase layer around the droplet, which increase mechanical resistance of the membrane layer and prevent coalescence of the dispersed droplets. This is supported by Okazaki [34] and Jilska and Geoff [35] who observed that more stable emulsion is obtained when volume fraction of organic phase is increased. On the other hand, 2.5, 3.9, and $4.0 \mu \mathrm{m}$ of droplet size was recorded for $1: 1$, $2: 1$, and $3: 1$ ratio respectively, indicating the size is within the range 


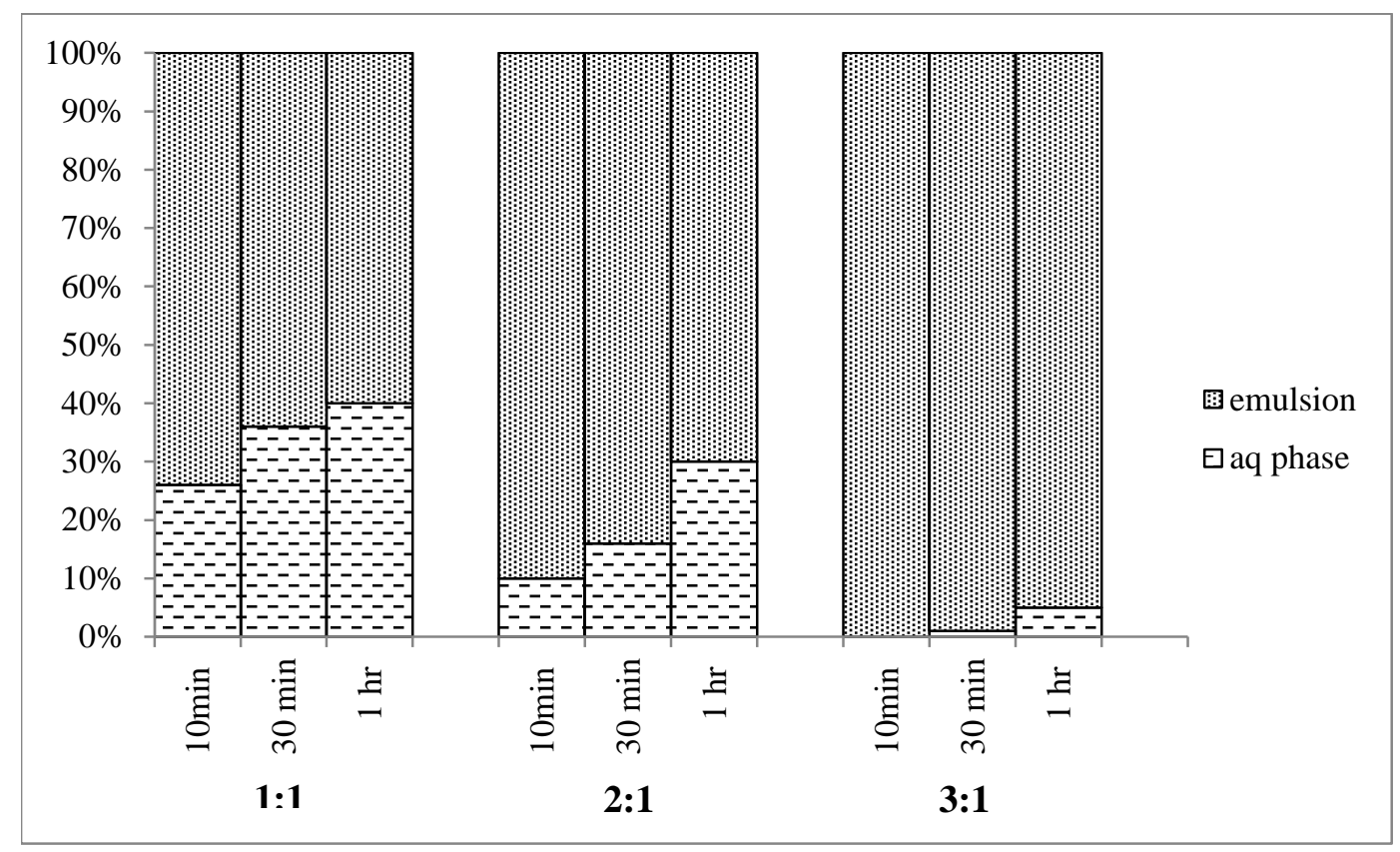

Figure 1 Effect of organic phase to internal phase ratio on primary emulsion stability [diluent: palm oil; [Amberlite LA2]: 0.05M; [Na2CO3]: 0.5M; speed: $5000 \mathrm{rpm}$; homogenizing time: $5 \mathrm{~min}$; Span $80: 3 \% \mathrm{w} / \mathrm{v}$; HLB: 4.3 ]

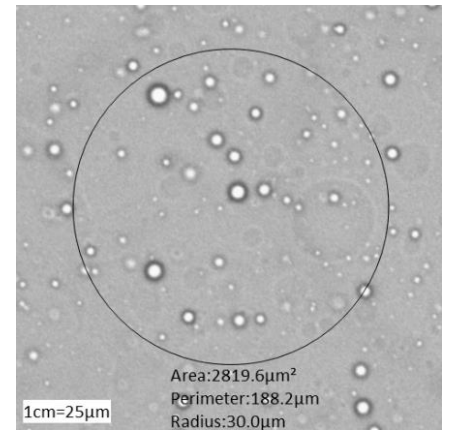

$1: 1$

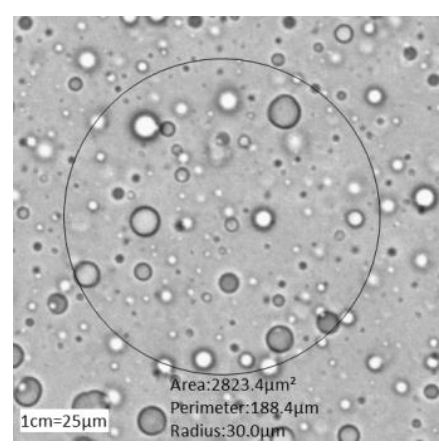

$2: 1$

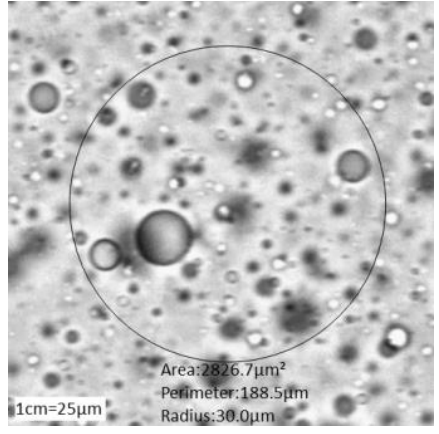

$3: 1$

Figure 2 Microscopic images of primary emulsion at different organic phase to internal phase ratio (O:I) (Magnification rate X400)

of standard droplet size [36]. In general, the larger droplet will increase the emulsion instability because the droplet easy to coalesce. However, in this study the largest droplet was observed at the most stable condition of 3:1 ratio, indicating the stability not necessarily depend on the droplet size. Hence, $3: 1$ of $\mathrm{O}$ : I ratio is highly preferable to produce a stable emulsion.

\subsubsection{Effect of Homogenizer Speed}

Suitable energy is required to form a stable emulsion. The effect of homogenizer speed on the emulsion stability was studied and the result is shown in Figure 3. Increasing homogenizer speed from 5000 to 7000 rpm increases the emulsion stability.

This is because higher homogenizer speed provide greater energy to expand the water-oil interface and generates more aqueous droplets, thus stabilize the emulsion. Several studies also reported higher speed increase emulsion stability [37-39]. Droplet count from Figure 4 proved that more droplets formed at $7000 \mathrm{rpm}$ compared to $5000 \mathrm{rpm}$. Further increase the 
speed to $9000 \mathrm{rpm}$ increase the number of droplet formed. However, Figure 3 shows that stability at $9000 \mathrm{rpm}$ is lower than that of $7000 \mathrm{rpm}$.

Rapid mixing causes the droplet tends to coalesce among each other, thus enlarging their size. Larger droplet size tends to settle faster than small droplet, hence destabilize the emulsion. Further increase homogenizer speed to $12000 \mathrm{rpm}$ formed a highly viscous, "mayonnaise-

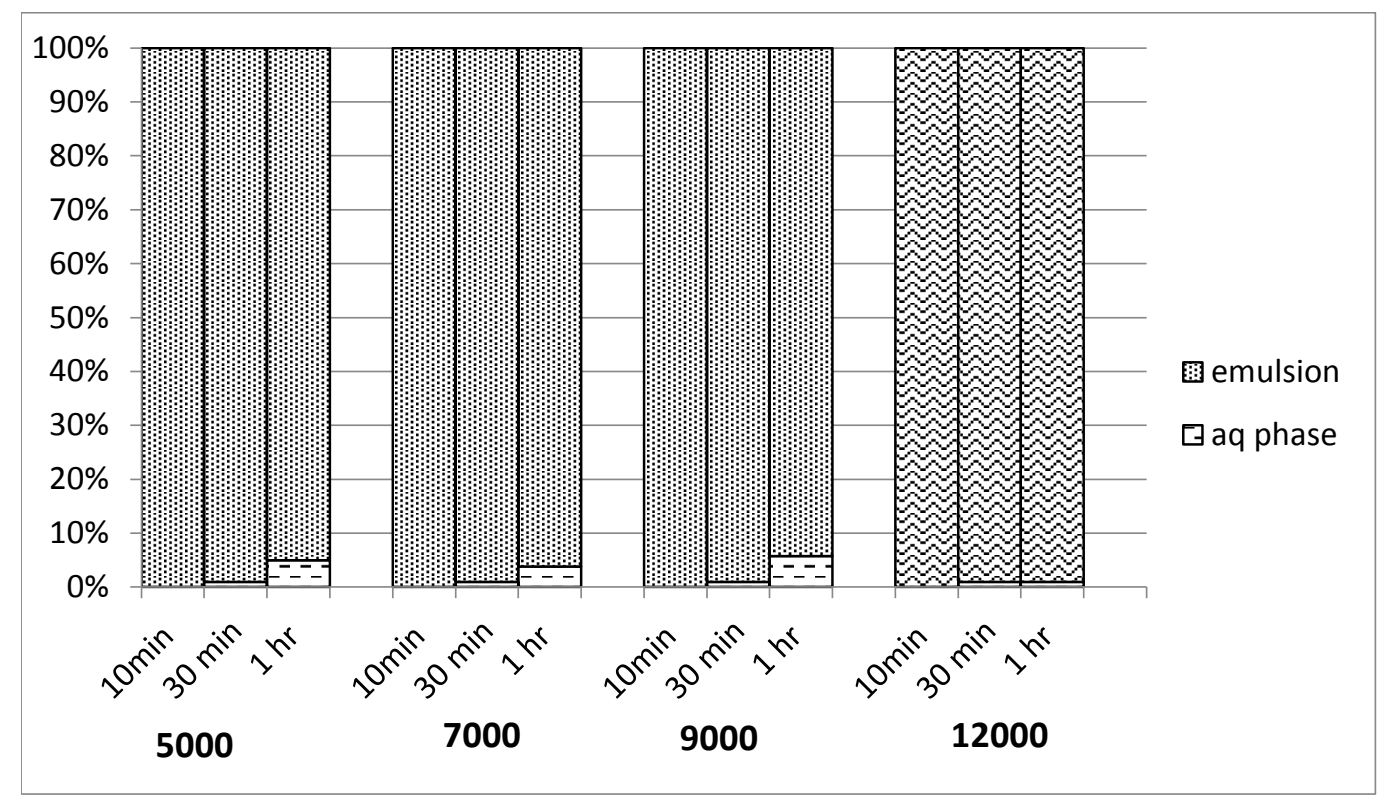

Figure 3 Effect of homogenizer speed on primary emulsion stability [diluent: palm oil; [Amberlite LA2]: 0.05M; [ $\mathrm{Na}_{2} \mathrm{CO}_{3}$ ]: 0.5M; O/I: 3/1; homogenizing time: 5min; Span 80: $3 \% \mathrm{w} / \mathrm{v} ; \mathrm{HLB}: 4.3]$
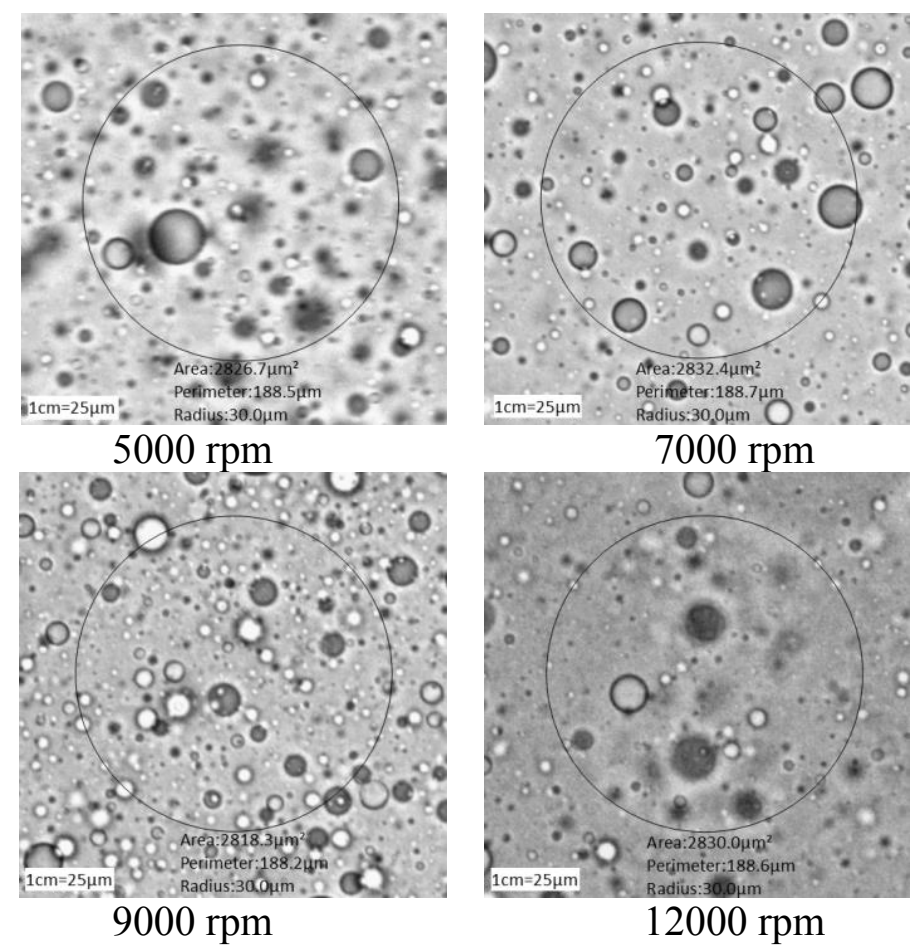
$7000 \mathrm{rpm}$

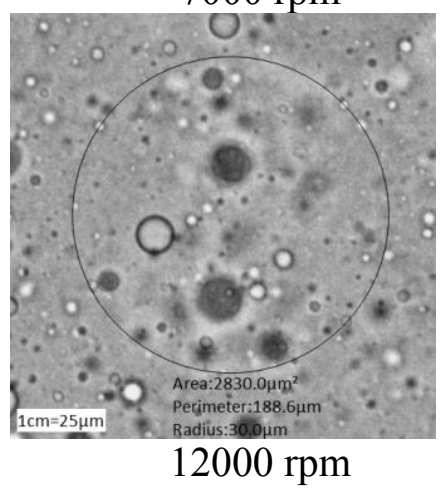

Figure 4 Microscopic images of primary emulsion for different homogenizer speed (Magnification rate $\mathrm{X} 400$ ) 
like" emulsion. This is due to forming mechanism at high speed, where airbubbles are merged into emulsion phase, resulting in a rigid system. Moreover, droplet formation also drops, indicating high shear exposure damage the droplet. The observation agrees with Sanna and Rose [30], who found high speed form highly viscous emulsion. Therefore, $7000 \mathrm{rpm}$ homogenizer speed is chosen for producing more stable emulsion.

\subsubsection{Effect of Homogenizing Time}

The effect of homogenizing time on the primary emulsion stability is demonstrated in Figure 5. The result shows that at 3 minutes emulsification time, about $2 \%$ of aqueous phase was separated from the emulsion within 10 minutes. This indicates that short emulsifying time produce unstable emulsion because of the mixture of organic membrane and aqueous internal solution was not well homogenized.

As a result, the configuration of Span 80 at the interfacial area is unorganized, less interfacial tension is reduced, and thus larger droplets were formed which is around $11.2 \mu \mathrm{m}$. At 5 minutes homogenizing time, the emulsion formed is more stable, where it starts to break after 30 minutes. The intensity of the solution is enhanced by longer homogenizing time. The emulsion will be more homogeneous with more internal phase entrapped in the membrane phase. Thus, smaller internal droplets were formed which is $4.9 \mu \mathrm{m}$ in size as shown in Figure 6 . However, further increase the emulsification time up to 10 minutes and above, unstable water-in-oil emulsion was formed. It is due to the emulsion was exposed to the longer time of high shear and causing emulsion breakage. In addition, the effectiveness of the surfactant also decreased due to the intense emulsification and caused the surfactant to drop out from the wateroil interface. This increase the interfacial tension and form larger droplet which is $8.3 \mu \mathrm{m}$ and easy to break. Other studies also supported breakage phenomena caused by prolonged emulsification time [40, 41].

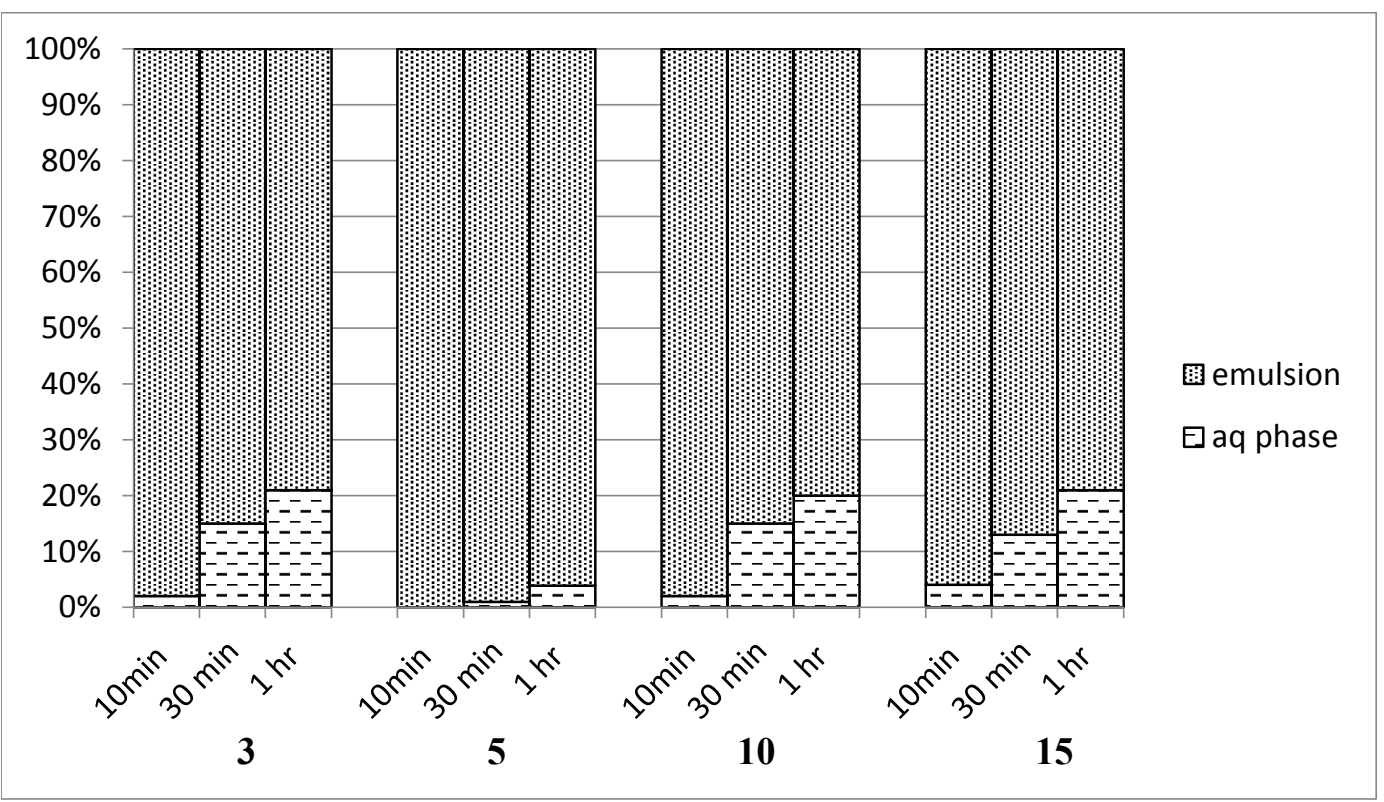

Figure 5 Effect of homogenizing time on primary emulsion stability [diluent: palm oil; [Amberlite LA2]: 0.05M; [ $\mathrm{Na}_{2} \mathrm{CO}_{3}$ ]:0.5M; O/I: 3/1; homogenizer speed: 7000; Span 80: 3\% w/v; HLB: $4.315 \mathrm{~min}$; Span 80: 3\% w/v; HLB: 4.31 

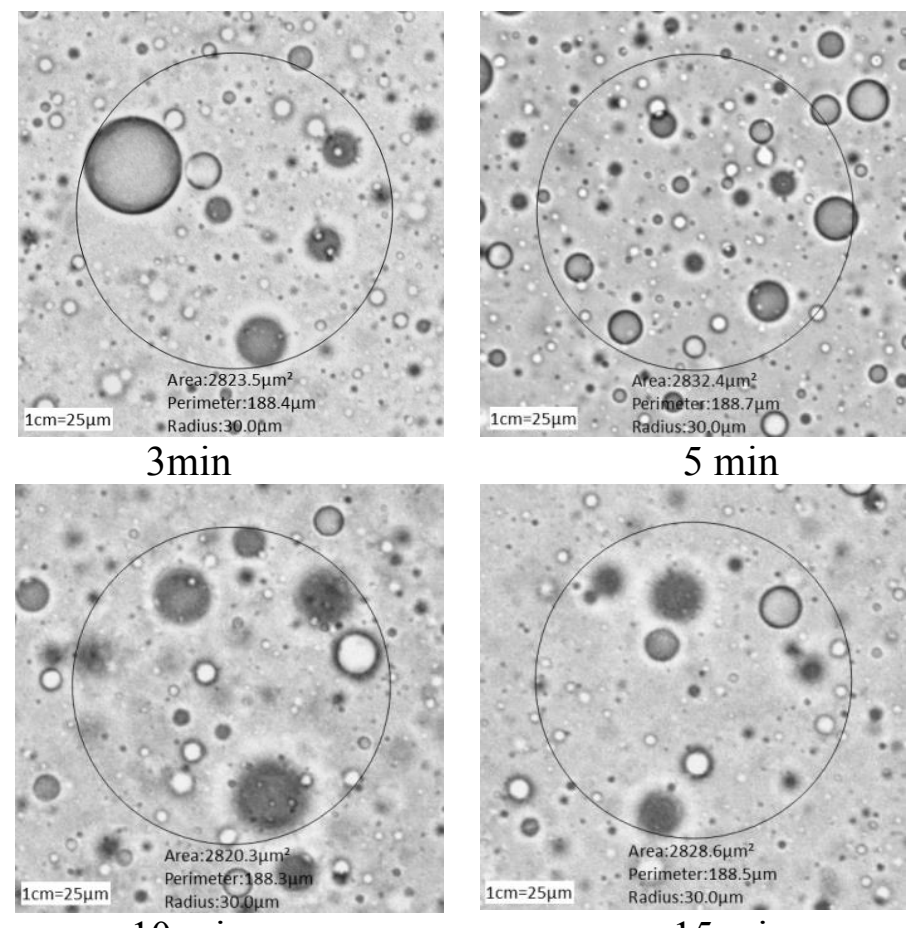

$10 \mathrm{~min}$

$15 \mathrm{~min}$

Figure 6 Microscopic images of primary emulsion at different homogenizing time (minutes) (Magnification rate X400)

\subsubsection{Effect of Surfactant Concentration}

The effect of varying surfactant concentration on the stability performance of water-in-oil emulsion was shown in Figure 7 . At $1 \%(\mathrm{w} / \mathrm{v})$ Span 80, aqueous phase begin to separate in the first 10 minutes, indicating low surfactant concentration is insufficient to reduce water-oil interfacial tension. This condition does not facilitate emulsion formation, where only 70 droplets were counted in the circle range of Figure 8. Higher interfacial tension cause immediate emulsion breakage. Increase the surfactant concentration to $3 \%$ enhanced emulsion stability, where only $1 \%$ of aqueous phase was separated after 30 minutes. At higher surfactant concentration, more surfactant adsorbs at the interface between the oil membrane phase and internal phase, thus enhances the strength of adsorption layer and increase stability. Figure 8 shows greater number of droplets formed, which is 150 droplets, representing sufficient surfactant was added into the system. Increasing surfactant concentration to 5 and $7 \%$ resulted in unstable emulsion. At high surfactant concentration destabilization of emulsion occur from rapid coalescence between droplets. Therefore, less droplets was counted for $5 \%$ and $7 \%$ surfactant concentration which are 80 and 60 droplets respectively. A study by Joshi et al. [42] also observed destabilization of emulsion at higher surfactant. Further increase surfactant concentration to $10 \%$ resulted in highly viscous "mayonnaise-like" emulsion. This is due to the increasing viscosity of the membrane phase as tabulated in Table 2. At high viscosity, mechanical energy provided is insufficient to expand the interface, thus forming highly viscous emulsion. Aside from instability, high surfactant concentration also not favorable 


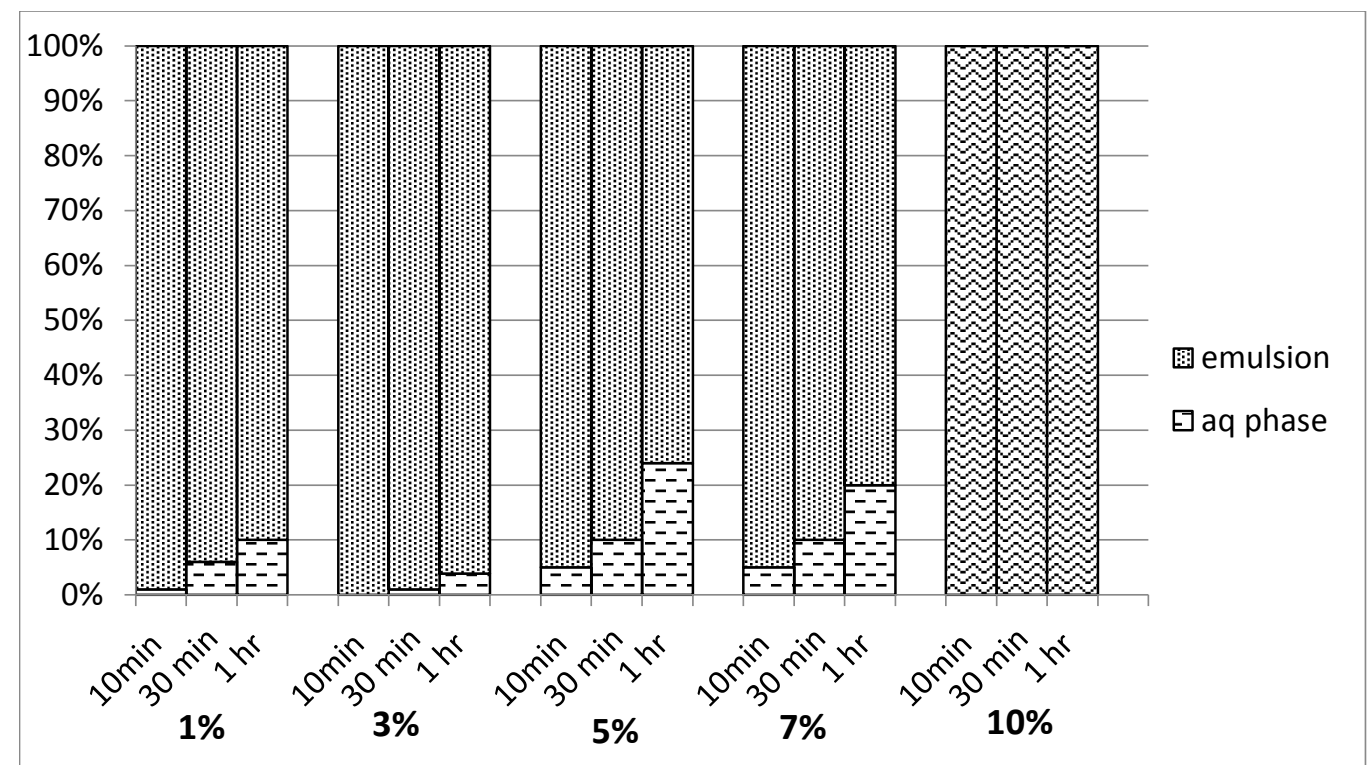

Figure 7 Effect of surfactant concentration on primary emulsion stability [diluent: palm oil; [Amberlite LA2]: 0.05M; $\left[\mathrm{Na}_{2} \mathrm{CO}_{3}\right]: 0.5 \mathrm{M} ; \mathrm{O} / \mathrm{I}: 3 / 1$; homogenizer speed: 7000 ; homogenizing time: $5 \mathrm{~min}$; HLB: 4.31
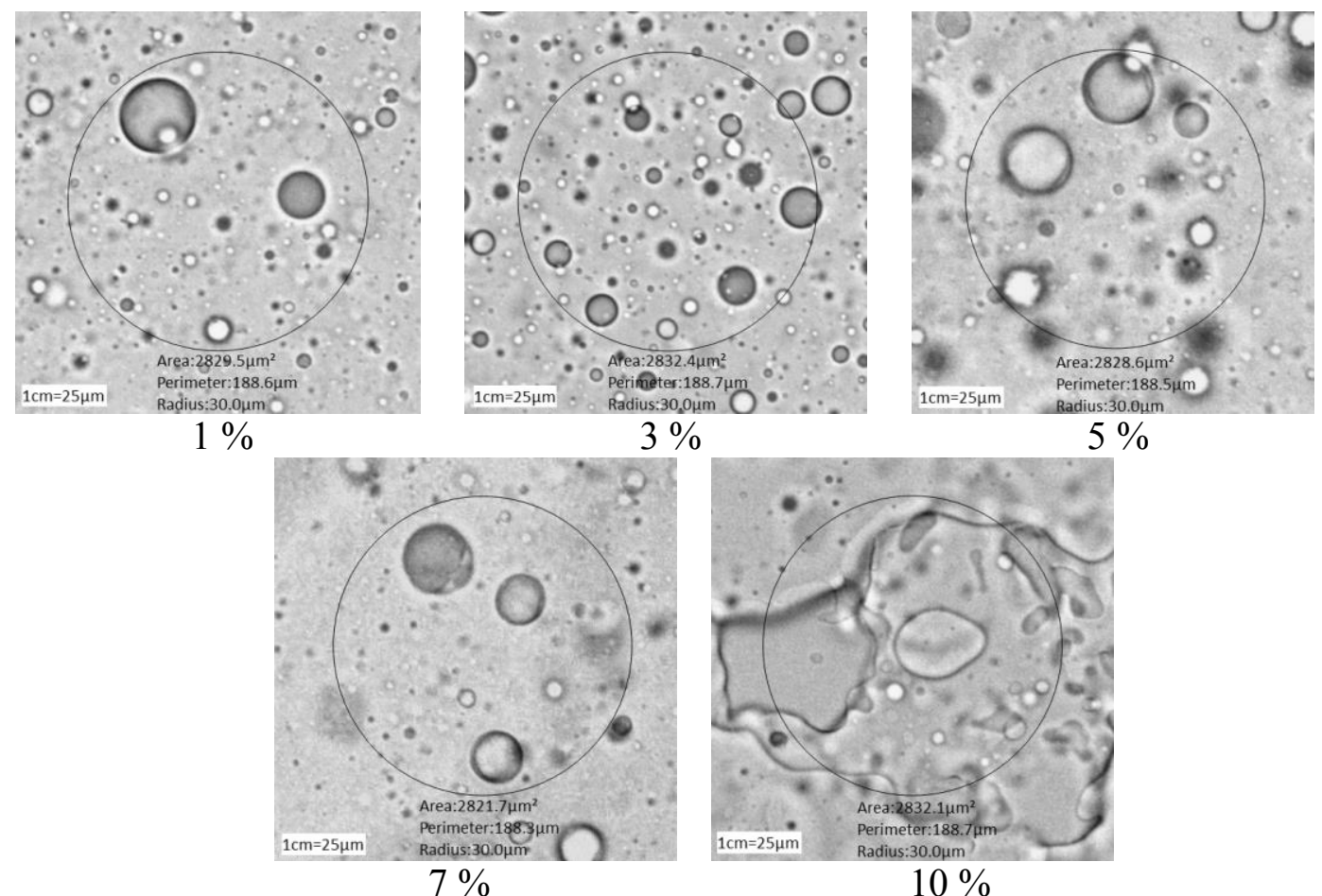

Figure 8 Microscopic images of primary emulsion for different surfactant concentration $(\% \mathrm{w} / \mathrm{v})$ (Magnification rate $\mathrm{X} 400)$

because it can hinder mass transfer solute by increasing the interfacial resistance and interfere with the carrier reaction at the interface [40]. Therefore, $3 \%(\mathrm{w} / \mathrm{v})$ surfactant concentration was sufficient to form stable emulsion.
Table 2 Viscosity of liquid membrane at different surfactant concentration

\begin{tabular}{cc}
\hline Span80 (\%w/v) & Viscosity (cp) \\
\hline 3 & 48.26 \\
5 & 53.33 \\
7 & 55.15 \\
10 & 59.39 \\
\hline
\end{tabular}




\subsubsection{Effect of Surfactant Blend}

Mixtures of emulsifiers can improve stability of emulsions. The efficiency of surfactant combination (usually of low and high HLB) leads to a greatly enhanced stability as compared to individual emulsifiers [29, 38]. Basically, the addition of a cosurfactant can further reduce interfacial tension, also through adsorbing in the water-in-oil interface. It thus minimizes the repulsion of the hydrophilic head-groups of the surfactants, which contributes to a more efficient packing of the surfactants at the interface and promote droplet formation. In this study, the result of emulsion stability at different composition of Span and Tween 80 is presented in Figure 9. The surfactant composition was represented by hydrophilic-lipophilic balance (HLB) values. Increasing the HLB value from 4.3 to 6 reduces the emulsion stability. This is because the amount of Tween 80 added is not enough to form a mixed surfactant layer and cause irregular arrangement of the surfactant at the interface. Hence, the amount of droplet formed was reduced from 150 to 80 droplets as can be seen in Figure 10. Further increase the HLB value at 8 causes the emulsion become more

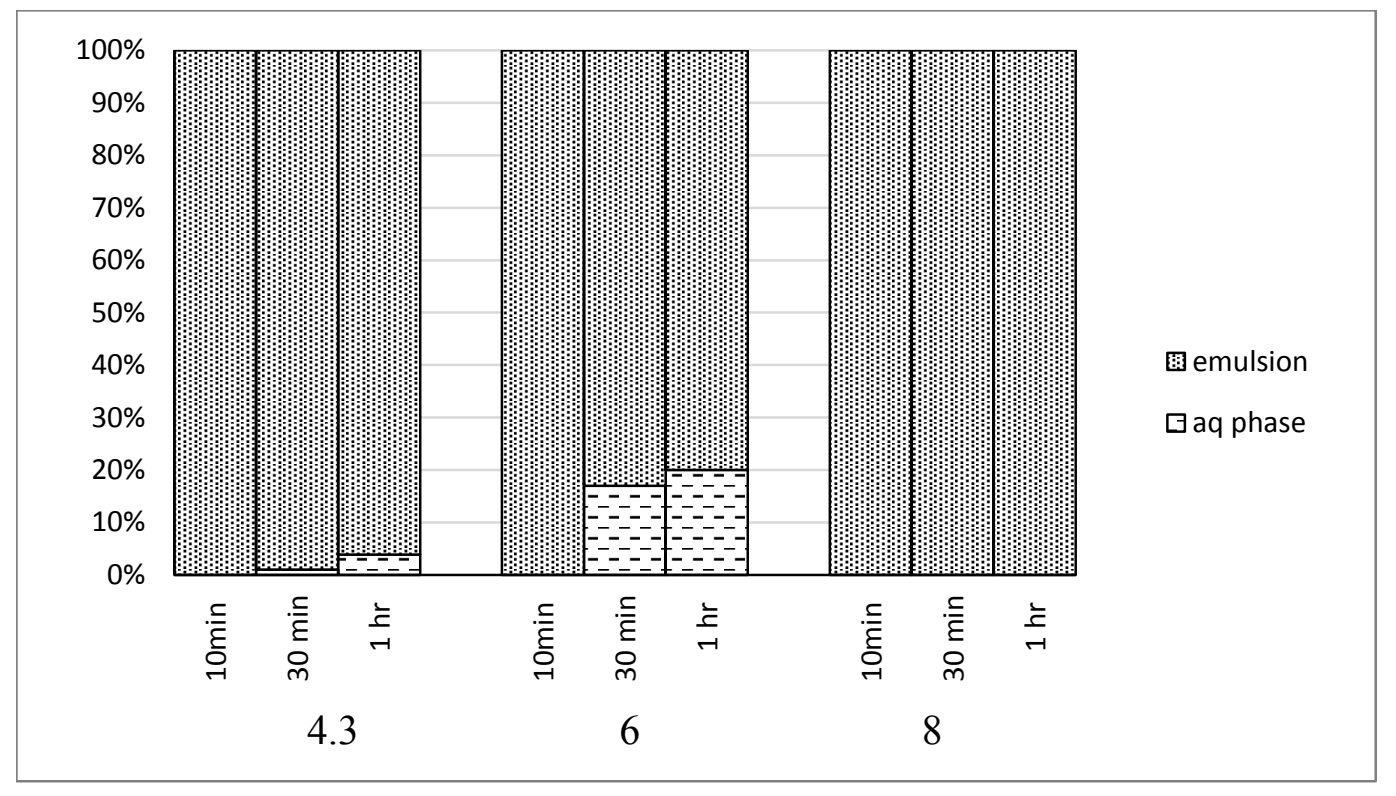

Figure 9 Effect of surfactant blend on primary emulsion stability at different HLB [diluent: palm oil; [Amberlite LA2]: 0.05M; $\left[\mathrm{Na}_{2} \mathrm{CO}_{3}\right.$ ]:0.5M; O/I: 3/1; homogenizer speed: 7000; homogenizing time: $5 \mathrm{~min}$; surfactant concentration: $3 \% \mathrm{w} / \mathrm{v}$ ]

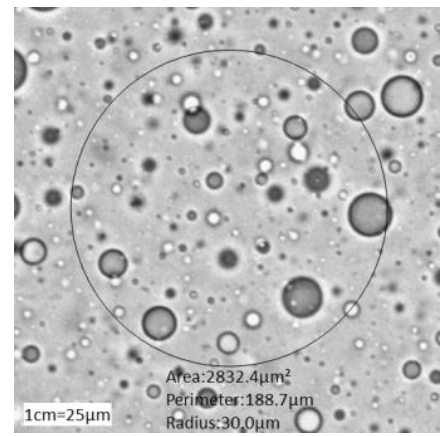

HLB 4.3 (3\% Span

80)

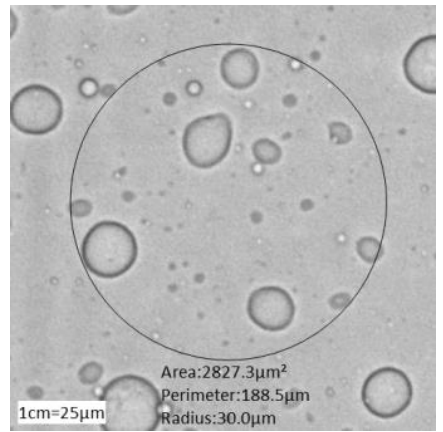

HLB $6(2.5 \%$ Span $80+$

$0.5 \%$ Tween 80 )

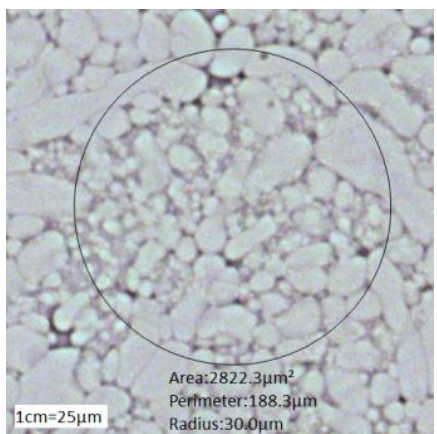

HLB $8(2 \%$ Span $80+$

$1 \%$ Tween 80 )

Figure 10 Microscopic images of primary emulsion for different HLB value (Magnification rate X400) 
stable. This indicates that Span 80 is compatible with Tween 80 due to similarity structure between both surfactants, since Tween 80 is a derivative from Span 80. Other than that, this situation has caused the film of one surfactant be better solvent for the second surfactant on the mixed film and formed a phase that resists collapse, making the emulsion more stable. The result show that no phase separation occurred for HLB 8. Thus, the combination of Span 80 and Tween 80 at HLB 8 will be considered for the extraction study.

\subsection{ELM Extraction Study}

\subsubsection{Transport Mechanism of Carboxylic Acid}

Mechanism of carrier-facilitated transport extraction and stripping of succinic acid by Amberlite LA2 is schematically presented in Figure 11. The complexation reaction between undissociated carboxylic acid $\left(\mathrm{H}_{2} \mathrm{~A} 2\right)$ with Amberlite LA2 (B) can be expressed by $[43,44,24]$ :

$$
\begin{aligned}
\left(H_{2} A\right)(\text { aq. }) & +y B(\text { org. }) \\
& \leftrightarrow\left(H_{2} A\right) B(\text { org. })
\end{aligned}
$$

The reaction involved in the transport of the succinic acid by Amberlite LA2 is an acid-base reaction. At the external interface, succinic acid form complex with Amberlite LA2. The complex then transported through the membrane phase to the internal interface between the membrane phase and internal striping phase. At the internal interface, sodium carbonate react with the complex, regenerating Amberlite LA2 in the membrane phase and the product in the internal phase is the acid in the form of sodium salt, and carbonic acid as secondary product. Mass transfer in this system is governed by the carrier present in the membrane phase as well as $\mathrm{pH}$ and ion concentration gradient between external and internal aqueous phase.

\subsubsection{Effect of Stripping Agent Concentration}

The performance of $\mathrm{Na}_{2} \mathrm{CO}_{3}$ as stripping agent is illustrated in Figure 12. The result shows that increasing $\mathrm{Na}_{2} \mathrm{CO}_{3}$ concentration from $0.005 \mathrm{M}$ to $0.01 \mathrm{M}$ increase the extraction from $55 \%$ and $63 \%$ respectively. This indicates larger reaction capacity of $\mathrm{Na}_{2} \mathrm{CO}_{3}$ with succinic acid at higher $\mathrm{Na}_{2} \mathrm{CO}_{3}$ concentration. In addition, higher $\mathrm{Na}_{2} \mathrm{CO}_{3}$ concentration increase the tendency of the internal phase to strip out the solute, which avoid the

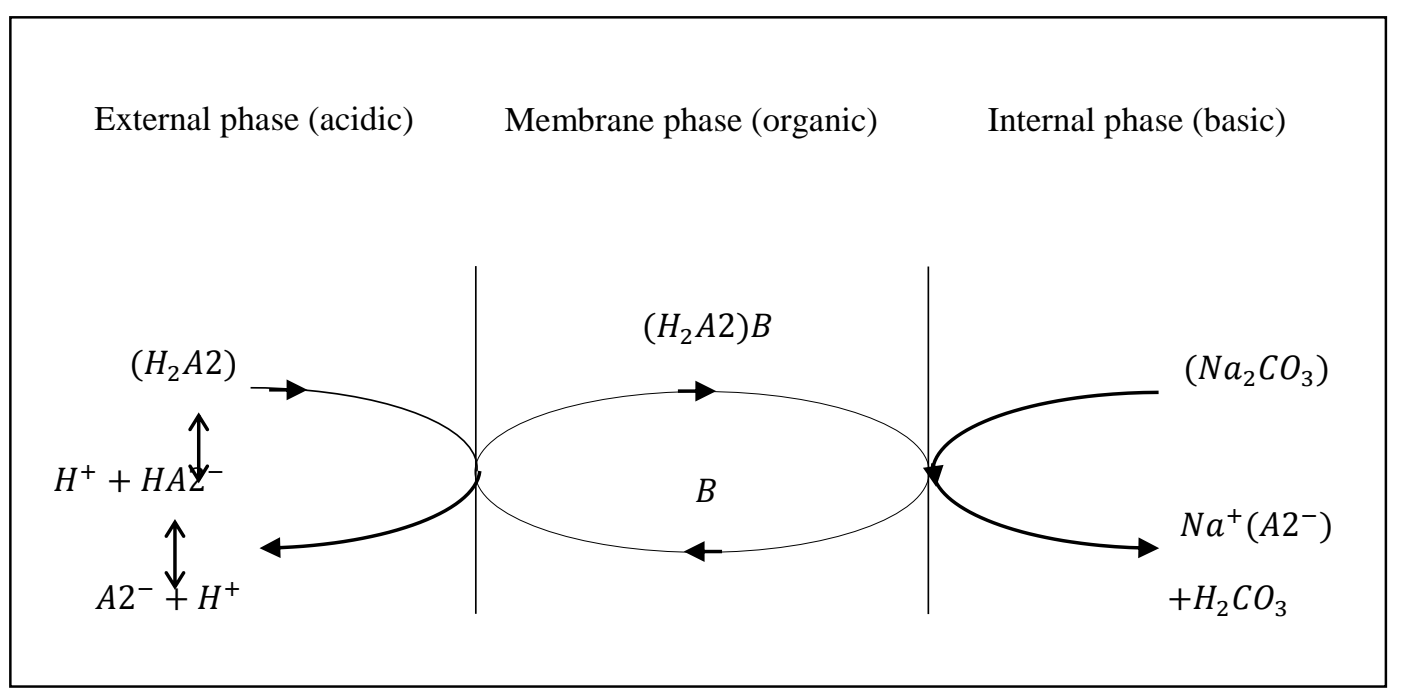

Figure 11 Transport mechanism for extraction of succinic acid and by ELM $\left(H_{2} A 2\right.$ : succinic acid, $B$ : amberlite LA-2) 
accumulation of succinic-carrier complex in the membrane layer, thus increase the extraction. However, further increase the concentration up to $0.05 \mathrm{M}$ and $0.1 \mathrm{M}$, the extraction of succinic acid reduced to 56 and $49 \%$. It is due to the higher concentration of $\mathrm{Na}_{2} \mathrm{CO}_{3}$ creates very large concentration gradient with the external phase and cause the internal droplet to break. As a result, the succinic acid stripped into the internal phase leak to the external phase and lowers the extraction performance. Thus, $0.01 \mathrm{M} \mathrm{Na}_{2} \mathrm{CO}_{3}$ was selected as the best stripping agent concentration in this process.

\subsubsection{Effect of Treat Ratio}

The effect of treat ratio which is initial volume ratio of emulsion to feed phase on succinic acid extraction is presented in Figure 13. At 1:1 treat ratio, the extraction of succinic acid is $58 \%$. The extraction is lower due to the osmosis pressure effect which reduce the globule formation, thus reduce the mass transfer area available for the extraction. Besides, lower extraction is also due to the breakage of water-in-oil emulsion. From the observation, the globules were formed initially when the primary emulsion was added into the feed phase. However, the emulsion is not fully dispersed into the external phase and starts to coalesce. This is due to the attractive force between the globules [44]. Increasing the treat ratio to $1: 3$ increases the percentage of extraction to $63 \%$. This is because the osmotic pressure effect is reduced at higher treat ratio, therefore generates more globules and promotes better succinic acid extraction. Further increase the treat ratio to $1: 5$ and 1:7, the percentage of extraction decrease to 51 and $40 \%$ respectively. Basically, the volume of internal solution towards external phase is decreases at fix emulsion volume. Therefore, number of available interfacial surface area per unit volume of external solution decreases. Thus, it reduces the mass transfer of succinic acid from the external to the internal phase. Therefore, 1:3 treat ratio was selected in this study.

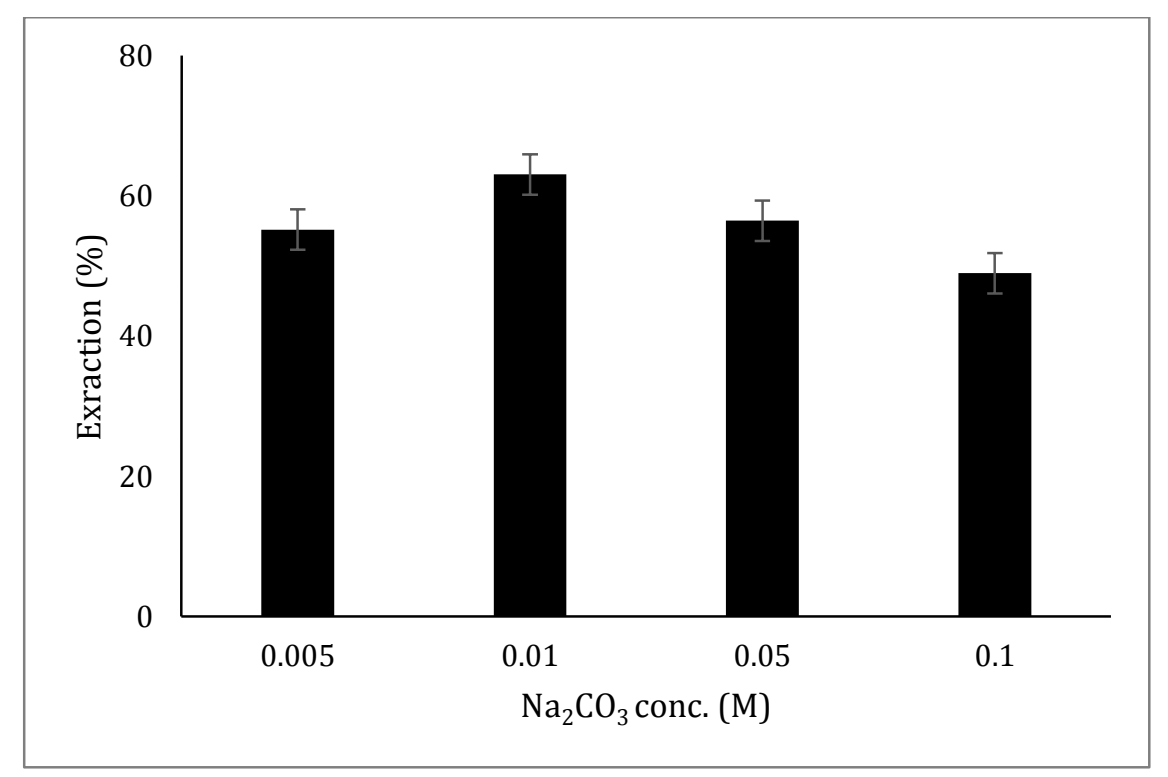

Figure 12 Effect of $\mathrm{Na}_{2} \mathrm{CO}_{3}$ concentration towards the performance of succinic acid extraction [agitation speed: $300 \mathrm{rpm}$, [octanol]: $10 \%$, [Amberlite LA2]: $0.5 \mathrm{M}$, treat ratio: $1: 3]$ 


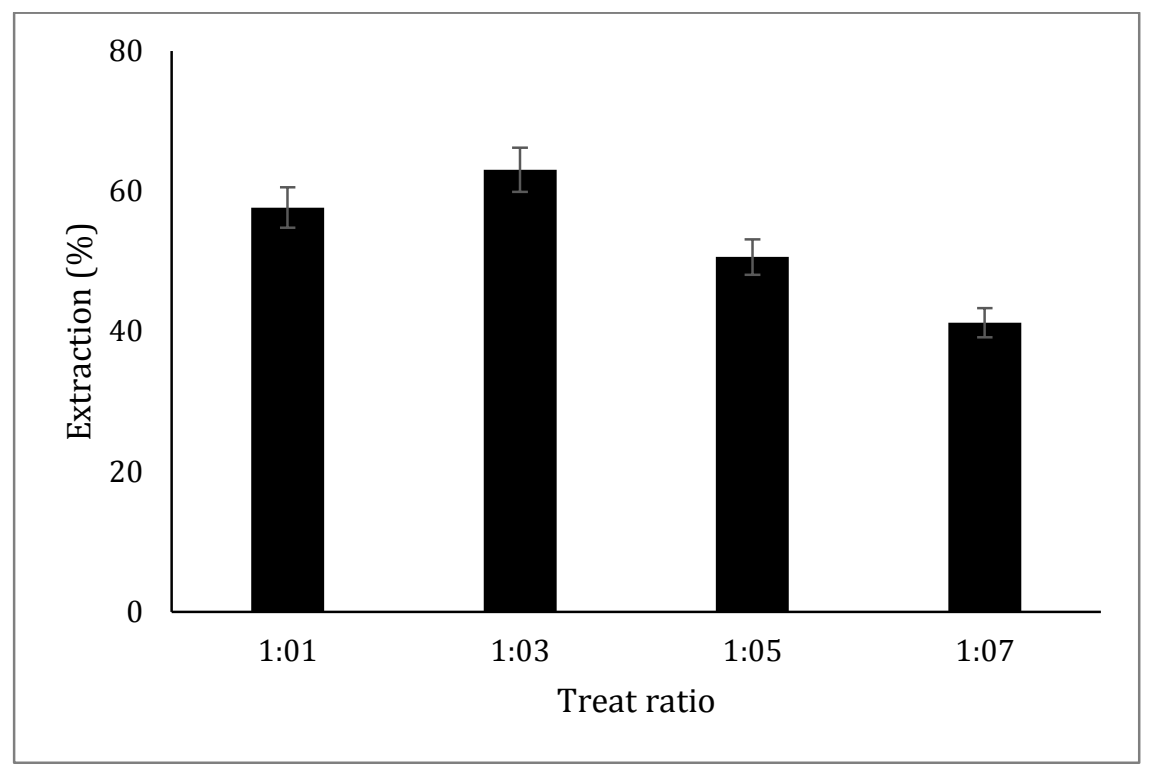

Figure 13 Effect of treat ratio towards the performance of succinic acid extraction [agitation speed: 300rpm, [octanol]: 10\%, [Amberlite LA2]: 0.5M, [ $\mathrm{Na}_{2} \mathrm{CO}_{3}$ ]: $0.01 \mathrm{M}$ ]

\subsubsection{Effect of Carrier Concentration}

The effect of Amberlite LA2 concentration on the extraction of succinic acid is shown in Figure 14. The extraction of succinic acid increase from $57 \%$ to $71 \%$ when the concentration of Amberlite LA2 increased from 0.005 to $0.05 \mathrm{M}$ respectively. It indicates that Amberlite LA2 plays the role of carrier for succinic acid extraction as shown in Figure 11. Amberlite LA-2 and succinic acid reacts reversibly to form a succinic-amine complex at the external interface between the external feed and the membrane phases. The

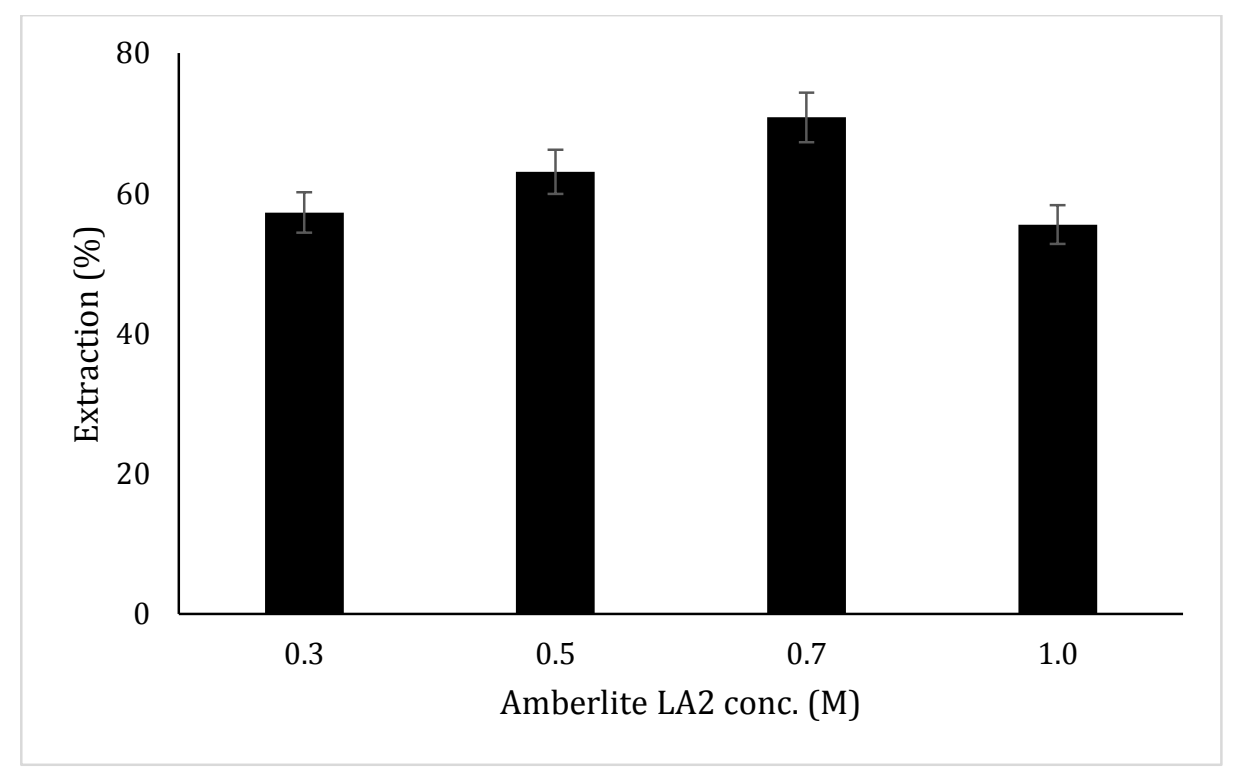

Figure 14 Effect of Amberlite LA2 concentration towards the performance of succinic acid extraction [agitation speed: $300 \mathrm{rpm}$, [octanol]: $10 \%,\left[\mathrm{Na}_{2} \mathrm{CO}_{3}\right]: 0.01 \mathrm{M}$, treat ratio: 1:3 
concentration gradient of the complex across the membrane will increase the flux of succinic acid through the membrane. Thus, the degree of extraction of succinic acid increased with an increase in Amberlite LA-2 concentration. This is supported by Lee [45] who reported succinic acid extraction efficiency increased when increasing Amberlite LA2 concentration. Further increase Amberlite LA2 concentration to $1.0 \mathrm{M}$, the succinic acid extraction decrease to $55 \%$. It is due to higher Amberlite LA2 concentration also increase the viscosity of the membrane. This attribute to the formation of larger emulsion globule and reduce mass transfer area for the ELM extraction process. This is in accordance with the study by Pawel and Piotr [46], Sulaiman et al. [39], and Lee [45], who found that the membrane viscosity will increase as the carrier concentration is increased. Hence, $0.7 \mathrm{M}$ Amberlite LA2 was chosen as optimum carrier in ELM extraction of succinic acid.

\subsection{CONCLUSION}

Stability of primary emulsion play an important role in ELM process. In this study, the most stable emulsion was observed at $3: 1$ organic phase to internal phase ratio, $7000 \mathrm{rpm}$ homogenizer speed, 5 minute emulsification time, $3 \%(\mathrm{w} / \mathrm{v})$ of surfactant of HLB value of 8 with combination of Span 80 and Tween 80 . Meanwhile, up to $70 \%$ of succinic acid was extracted under favorable conditions, at $0.01 \mathrm{M} \mathrm{Na}_{2} \mathrm{CO}_{3}, 1: 3$ treat ratio, and $0.7 \mathrm{M}$ Amberlite LA2. Therefore, the formulated liquid membrane was stable and has high possibility to be used in the succinic acid purification process.

\section{ACKNOWLEDGEMENT}

The authors would like to acknowledge the Ministry of Higher Education (MOHE), Universiti Teknologi Malaysia (RU Research Grant; GUP:Q.J130000.2544.04H47), and Centre of Lipid Engineering and Applied Research (CLEAR) for financial support to make this research possible.

\section{REFERENCES}

[1] J. G. Zeikus, M. K. Jain, P. Elankovan. 1999. Biotechnology Of Succinic Acid Production And Markets For Derived Industrial Products. Appl. Microbiol. Biotechnol. 51: 545552.

[2] S. Varadarajan, D. Miller. 1999. Catalytic Upgrading Of Fermentation-Derived Organic Acids. Biotechnol. Progr. 15: 845-854.

[3] I. Bechthold, K. Bretz, S. Kabasci, R. Kopitzky, A. Springer. 2008. Succinic Acid: A New Platform Chemical For Biobased Polymers From Renewable Resources. Chem. Eng. Technol. 31: 647-654.

[4] C. Delhomme, D. Weuster-Botz, F. E. Kuhn. 2009. Succinic Acid From Renewable Resources As A C4 Building-Block ChemicalA Review Of The Catalytic Possibilities In Aqueous Media. Green Chem. 11: 13-26.

[5] K. K. Cheng, X. B. Zhao, J. Zeng, J. A. Zhang. 2012. Biotechnological Production Of Succinic Acid: Current State And Perspectives. Biofuels, Bioprod. Biorefin. 6: 302-318.

[6] C. Efe, M. Pieterse, L. A. M. van der Wielen, A. J. J. Straath of. 2011. Separation Of Succinic 
Acid From Its Salts On A HighSilica Zeolite Bed. Chem. Eng. Process. Process Intensif. 50: 1143-1151.

[7] A. Cukalovic, C. V. Stevens. 2008. Feasibility Of Production Methods For Succinic Acid Derivatives: A Marriage Of Renewable Resources And Chemical Technology. Biofuels, Bioprod. Biorefin. 2: 505-529.

[8] L. Liao, M. Zhao, H. Zhao, J. Ren, C. Cui, X. Hu. 2009. Effect Of Succinic Acid DeamidationInduced Modification On Wheat Gluten. Front. Chem. Eng. China. 3: 386-392.

[9] P. C. Lee, S. Y. Lee, S. H. Hong, H. N. Chang, S. C. Park. 2003. Biological Conversion Of Wood Hydrolysate To Succinic Acid By Anaerobiospirillum Succiniciproducens. Biotechnol. Lett. 25: 111-114.

[10] C. Andersson, D. Hodge, K.A. Berglund, U. Rova. 2007. Effect Of Different Carbon Sources On The Production Of Succinic Acid Using Metabolically Engineered Escherichia Coli. Biotechnol. Progr. 23: 381-388.

[11] Y. P. Liu, P. Zheng, Z. H. Sun, Y. Ni, J. J. Dong, P. Wei. 2008. Strategies Of $\mathrm{Ph}$ Control And Glucose-Fed Batch Fermentation For Production Of Succinic Acid By Actinobacillus Succinogenes CGMCC1593. J. Chem. Technol. Biotechnol. 83: 722-729.

[12] P. Zheng, L. Fang, Y. Xu, J. J. Dong, Y. Ni, Z. H. Sun. 2010. Succinic Acid Production From Corn Stover By Simultaneous Saccharification And Fermentation Using Actinobacillus Succinogenes. Bioresour. Technol. 101: 78897894.

[13] C. C. J. Leung, A. S. Y. Cheung, A. Y. Z. Zhang, K. F. Lam, C. S.
K. Lin. 2012. Utilisation Of Waste Bread For Fermentative Succinic Acid Production. Biochem. Eng. J. 65: 10-15.

[14] T. Willke, K. D. Vorlop. 2004. Industrial Bioconversion of Renewable Resources As An Alternative To Conventional Chemistry. Appl. Microbiol. Biotechnol. 66: 131-142.

[15] R. Datta, D. A. Glassner, M. K. Jain, J. R. Vick Roy. 1992. US Patent, 5168055.

[16] R. Luque, C. S. K. Lin, C. Du, D. J. Macquarrie, A. Koutinas, R. Wang, C. Webb, J. H. Clark. 2009. Chemical Transformations Of Succinic Acid Recovered From Fermentation Broths By A Novel Direct Vacuum Distillation-Crystallisation Method. Green Chem. 11: 193200.

[17] H. Wu, M. Jiang, P. Wei, D. Lei, Z. Yao, P. Zuo. 2011. CN Patent. 200910025531.5.

[18] Y. Hong, W. Hong, D. Han. 2001. Application Of Reactive Extraction To Recovery Of Carboxylic Acids. Biotechnol. Bioprocess Eng. 6: 386-394.

[19] T. Kurzrock, D. Weuster-Botz. 2011. New Reactive Extraction Systems For Separation Of BioSuccinic Acid. Bioprocess. Biosyst. Eng. 34: 779-787.

[20] X. Yang, Y. Zhang, S. Bao. 2016. Separation And Recovery Of Sulfuric Acid From Acidic Vanadium Leaching Solution Of Stone Coal Via Solvent Extraction. J. Environ. Chem. Eng. 4: 1399-1405.

[21] C. Schöller, J. B. Chaudhuri, D. L. Pyle. 1993. Emulsion Liquid Membrane Extraction Of Lactic Acid From Aqueous Solutions And Fermentation Broth. Biotechnol. Bioeng. 42: 50-58. 
[22] N. Othman, H. Mat, M. Goto. 2006. Separation Of Silver From Photographic Wastes By Emulsion Liquid Membrane System. J. Membr. Sci. 282: 171-177.

[23] R. Sabry, A. Hafez, M. Khedr, A. El-Hassanin. 2007. Removal Of Lead By An Emulsion Liquid Membrane: Part I. Desalination. 212: 165-175.

[24] S. C. Lee, K. S. Hyun. 2010. Development Of An Emulsion Liquid Membrane System For Separation Of Acetic Acid From Succinic Acid. J. Membr. Sci. 350: 333-339.

[25] Y. S. Ng, N. S. Jayakumar, M. A. Hashim. 2010. Performance Evaluation Of Organic Emulsion Liquid Membrane On Phenol Removal. J. Hazard. Mater. 184: 255-260.

[26] Z. Y. Ooi, N. Othman, N. F. Mohamed Noah. 2016. Response Surface Optimization Of Kraft Lignin Recovery From Pulping Wastewater Through Emulsion Liquid Membrane Process. Desalin. Water Treat. 57: 78237832.

[27] V. S. Kislik. 2010. Liquid Membranes. Amsterdam: Elsevier.

[28] N. Othman, S. N. Zailani, N. Mili. 2011. Recovery Of Synthetic Dye From Simulated Wastewater Using Emulsion Liquid Membrane Process Containing Tri-Dodecyl Amine As A Mobile Carrier. J. Hazard. Mater. 198: 103-112.

[29] M. C. Chow, C. C. Ho. 1996. Properties Of Palm-Oil-In-Water Emulsions: Effect Of Mixed Emulsifiers. J. Am. Oil Chem. Soc. 73: 47-53.

[30] S. Björkegren, R. Fassihi Karimi, A. Martinelli, N. S. Jayakumar, M. A. Hashim. 2015. A New
Emulsion Liquid Membrane Based On A Palm Oil For The Extraction Of Heavy Metals. Membranes. 5: 168-179.

[31] V. Badgujar, N. K. Rastogi. 2011. Extraction Of Phenol From Aqueous Effluent Using Triglycerides In Supported Liquid Membrane. Desalin. Water Treat. 36: 187-196.

[32] C. Andersson. 2009. Biobased Production Of Succinic Acid By Escherichia Coli Fermentation. Thesis for the Doctoral Degree. Sweden: Luleå University of Technology.

[33] D. J. McClements, S. R. Dungan, J. B. German, C. Simoneau, J. E. Kinsella. 1993. Droplet Size And Emulsifier Type Affect Crystallization And Melting Of Hydrocarbon- In- Water Emulsions. J. Food Sci. 58: 1148-1151.

[34] S. Okazaki, M. Imai, M. Shimizu. 1990. Proceedings of the International Solvent Extraction Conference ISEC'90. New York: Elsevier Science.

[35] K. P. Anil, S. H. R. Syed, M. S. Ana. 2008. Handbook of Membrane Separations. New York: CRC Press.

[36] W. S. Ho, T. A. Hatton, E. N. Lightfoot, N. N. Li. 1982. Batch Extraction With Liquid Surfactant Membranes: A Diffusion Controlled Model. AIChE J. 28: 662-670.

[37] N. Othman, H. Mat, M. Goto. 2005. Selective Extraction Of Silver From Liquid Photographic Waste. Solvent Extr. Res. Dev. 12: 27-34.

[38] T. F. Tadros. 2009. Emulsion Science and Technology. United Kingdom: Wiley-VCH Verlag $\mathrm{GmbH} \& \mathrm{Co}$. KGaA.

[39] R. N. R. Sulaiman, N. Othman, N. A. S. Amin. 2014. Emulsion 
Liquid Membrane Stability In The Extraction of Ionized Nanosilver From Wash Water. $J$. Ind. Eng. Chem. 20: 3243-3250.

[40] N. Othman, N. Mili, A. Idris, S. N. Zailani. 2012. Removal Of Dyes From Liquid Waste Solution: Study On Liquid Membrane Component Selection And Stability. New Jersey: John Wiley \& Sons, Inc.

[41] Z. Y. Ooi, N. Harruddin, N. Othman. 2015. Recovery Of Kraft Lignin From Pulping Wastewater Via Emulsion Liquid Membrane Process. Biotechnol. Progr. 31: 1305-1314.

[42] H. C. Joshi, I. P. Pandey, A. Kumar, N. A. Garg. 2012. A Study Of Various Factors Determining The Stability Of Molecules. Adv. Pure Appl. Chem. 1: 7-11.

[43] J. A. Tamada, A. S. Kertes, C. J. King. 1990. Extraction Of
Carboxylic Acids With Amine Extractants. 1. Equilibria And Law Of Mass Action Modeling Ind. Eng. Chem. Res. 29: 13191326.

[44] I. Inci. 2007. Linear Solvation Energy Relationship Modeling And Kinetic Studies On Reactive Extraction Of Succinic Acid By tridodecylamine dissolved in MIBK. Biotechnol. Progr. 23: 1171-1179.

[45] S. C. Lee. 2011. Extraction Of Succinic Acid From Simulated Media By Emulsion Liquid Membranes. J. Membr. Sci. 381: 237-243.

[46] P. Dzygiel, P. Wieczorek. 2000. Extraction Of Amino Acids With Emulsion Liquid Membranes Using Industrial Surfactants And Lecithin As Stabilizers. $J$. Membr. Sci. 172: 223-232. 